\title{
Hard X-Ray Photon-In-Photon-Out Spectroscopy with Lifetime Resolution - of XAS, XES, RIXSS and HERFD
}

\author{
P. Glatzel ${ }^{\mathrm{a}}$, M. Sikora ${ }^{\mathrm{a}}$, S. G. Eeckhout ${ }^{\mathrm{a}}$, O. V. Safonova ${ }^{\mathrm{a}}$, G. Smolentsev ${ }^{\mathrm{b}}$, G. \\ Pirngruber $^{c}$, J. A. van Bokhoven ${ }^{c}$, J.-D. Grunewaldt ${ }^{c}$, M. Tromp ${ }^{d}$ \\ ${ }^{a}$ European Synchrotron Radiation Facility (ESRF), BP22, 6 rue Jules Horowitz, 38043 Grenoble, France \\ ${ }^{b}$ Faculty of Physics, Rostov State University, Rostov-on-Don, Russia \\ ${ }^{c}$ Institute for Chemical and Bioengineering, ETH Zurich, 8093 Zurich, Switzerland \\ ${ }^{d}$ University of Southampton, School of Chemistry, Southampton, SO17 1BJ, United Kingdom
}

\begin{abstract}
Spectroscopic techniques that aim to resolve the electronic configuration and local coordination of a central atom by detecting inner-shell radiative decays following photoexcitation using hard X-rays are presented. The experimental setup requires an X-ray spectrometer based on perfect crystal Bragg optics. The possibilities arising from non-resonant (X-Ray Emission Spectroscopy - XES) and resonant excitation (Resonant Inelastic X-Ray Scattering Spectroscopy - RIXSS, High-Energy-Resolution Fluorescence Detected (HERFD) XAS) are discussed when the instrumental energy broadenings of the primary (beamline) monochromator and the crystal spectrometer for $\mathrm{X}$-ray emission detection are on the order of the core hole lifetimes of the intermediate and final electronic states. The small energy bandwidth in the emission detection yields line-sharpened absorption features. In transition metal compounds, electron-electron interactions as well as orbital splittings and fractional population can be revealed. Combination with EXAFS spectroscopy enables to extent the k-range beyond unwanted absorption edges in the sample that limit the EXAFS range in conventional absorption spectroscopy.
\end{abstract}

Keywords: Emission Spectroscopy, Coordination Chemistry, Electronic Structure, EXAFS PACS: 61.10.Ht, 87.64.Fb, 87.64.Gb, 71.70.Ch

\section{INTRODUCTION}

Photon-in-photon-out techniques are targeted at applications where the sample environment cannot be chosen freely, i.e. UHV conditions suitable for photoemission experiments are not possible, or when a bulk sensitive probe is desired. The preferred X-ray spectroscopy technique to study element specifically electronic structure and local coordination is X-ray absorption spectroscopy (XAS).[1,2] The near edge structure (XANES) is mainly used to obtain oxidation states, even though XANES also contains information on the local geometry and coordination. A detailed analysis of the XANES structure is a complex task because of the numerous interactions that contribute to its shape. The spectroscopy using the extended range (EXAFS) is well developed theoretically and experimentally but the technique has its inherent limitations (e.g. differentiation of elements close in atomic number Z) and the ideal experimental conditions (e.g. sample thickness, homogeneity) for a correct EXAFS analysis are not always given. It is thus desirable to introduce other techniques on the beamline that either provide a mean to verify the results obtained from XAS or yield additional information on the sample.

An X-ray spectrometer based on perfect crystal Bragg optics opens up new possibilities for X-ray spectroscopy. Detecting the emitted X-rays with an instrumental energy bandwidths on the order of the core hole lifetime broadening enables to resolve fine structure in the X-ray emission spectrum. This fine structure contains information on the electronic configuration and chemical environment of the emitting atom that is complementary to what can be obtained in XAS.[3] Such a secondary monochromator provides an additional tunable energy detection to the primary monochromator of the synchrotron radiation source. X-ray emission spectrosocpoy (XES) thus adds a dimension to XAS. The techniques arising from such an experimental setup have been named non-resonant XES, resonant XES or resonant inelastic X-ray scattering spectroscopy (RIXSS) and high-energy-resolution fluorescence

CP879, Synchrotron Radiation Instrumentation: Ninth International Conference, edited by Jae-Young Choi and Seungyu Rah

(C) 2007 American Institute of Physics 978-0-7354-0373-4/07/\$23.00 
detection (HERFD). They will be discussed here. Another technique, non-resonant X-ray Raman scattering, will be left out to be discussed by others.[4]

\section{X-RAY EMISSION DETECTION WITH LIFETIME RESOLUTION}

\section{Non-resonant XES}

With the excitation energy set well above $(>30 \mathrm{eV})$ an absorption edge the $\mathrm{X}$-ray emission spectrum is independent of the incident energy. The emitted $\mathrm{X}$-rays are generally referred to as fluorescence lines. The chemical dependence of the $K \beta$ lines in $3 d$ transition metals has been discussed by many authors.[3] The $K \beta_{1,3}$ lines reflect the valence shell spin state and thus enable to detect oxidation state changes as well as high-spin-low-spin transitions. The K $\beta$ satellite lines occur right below the Fermi level and thus show a very strong chemical dependence. In particular, they show a strong sensitivity to the type of ligand. Unlike XAS, the XES shows distinct signatures for different ligands that enable to identify the quality of the chemical environment much more exact than in XAS. For example, nitrogen and oxygen ligands can be clearly distinguished.[5] Also, ligand protonation yields spectral changes that can be assigned to e.g. $\mathrm{OH}^{-}$or $\mathrm{H}_{2} \mathrm{O}$ groups (Fig. 1). The spectral signature does not depend strongly on the bond distances in contrast to the X-ray absorption features. It is thus easier to assign a spectral feature to a change in the ligand environment. However, bond distances cannot be obtained accurately using non-resonant XES and the technique has thus to be seen as a complementary tool to EXAFS.

The K $\beta$ satellite spectral features can be calculated using the FEFF code or density functional theory. The latter has the advantage that the molecular orbitals can be visualized and assigned to spectral features. This opens a new realm for spectral interpretation that will help to understand the effect of changes in the ligand environment on the electronics structure. When interpreting the spectra it is important to be aware of possible multi-electron transitions. Their intensities depend on the incident energy and it is thus possible to identify them in the $\mathrm{K} \beta$ satellite spectrum.[6]
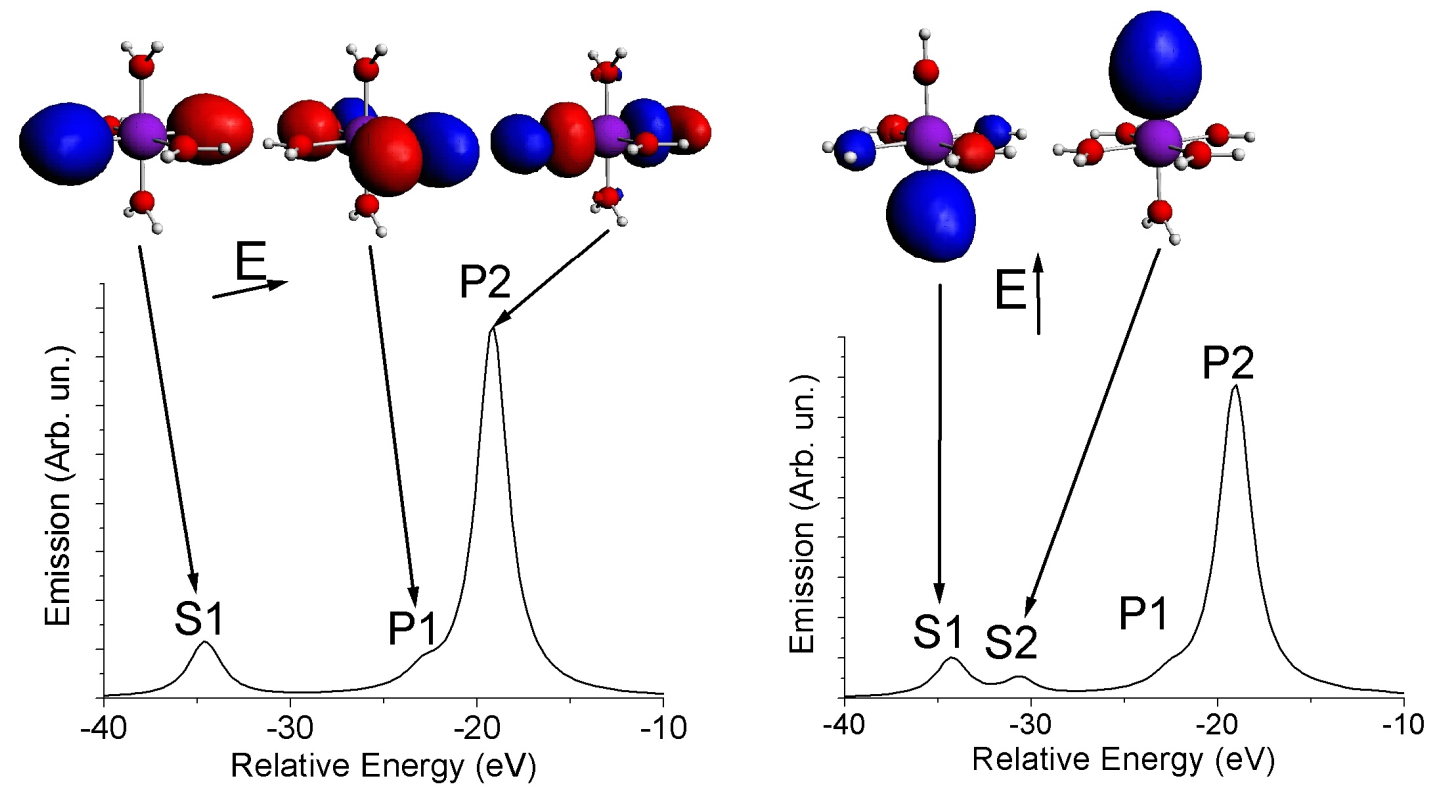

FIGURE 1. $\mathrm{K} \beta$ satellite lines for model systems $\left[\mathrm{Mn}\left(\mathrm{H}_{2} \mathrm{O}\right)_{6}\right]^{2+}$ (left) and $\left[\mathrm{Mn}\left(\mathrm{H}_{2} \mathrm{O}\right)_{5} \mathrm{OH}\right]^{+}$(right). Ligand deprotonation lowers the symmetry and yields an additional peak (S2) that can be assigned to a molecular orbital localized on the $\mathrm{OH}$ ligand. 


\section{HERFD XAS}

Fluorescence detected absorption spectroscopy was developed in order to be able to measure dilute samples that do not absorb enough photons to obtain a XAS spectrum in transmission mode. A XAS in fluorescence mode is detected either without energy resolution (e.g. photodiode) or with energy resolution (e.g. Ge detector). The better the energy resolution, i.e. the smaller the energy bandwidth around the fluorescence line, the better will be the signal to background ratio. The limit for improvement is set by the core hole lifetime broadening because a smaller bandwidth in the emission detection will not significantly improve the signal to background ratio anymore. We thus define high-energy-resolution fluorescence detected absorption spectroscopy (HERFD XAS) as detecting the fluorescence line with an energy bandwidth on the order or below the core hole lifetime broadening. Apart from the advantage of having the best possible signal to background ratio, emission detection with lifetime resolution also yields line-sharpened absorption features. This effect considerably helps to analyze absorption features. For 3d transition metals it has been used to separate the $\mathrm{K}$ absorption pre-edges from the strong main edge. Figure 2 shows that the effect is very pronounced for high-Z materials (e.g. Pt, Au, ...). The reduced line-broadening can be readily simulated in the FEFF code. The technique has been used to study catalysts under working conditions and it successfully helped to identify the bonding configuration of $\mathrm{CO}$ on Pt nano-particles or the actication of $\mathrm{O}_{2}$ over $\mathrm{Au}$ nano-particles.[7]
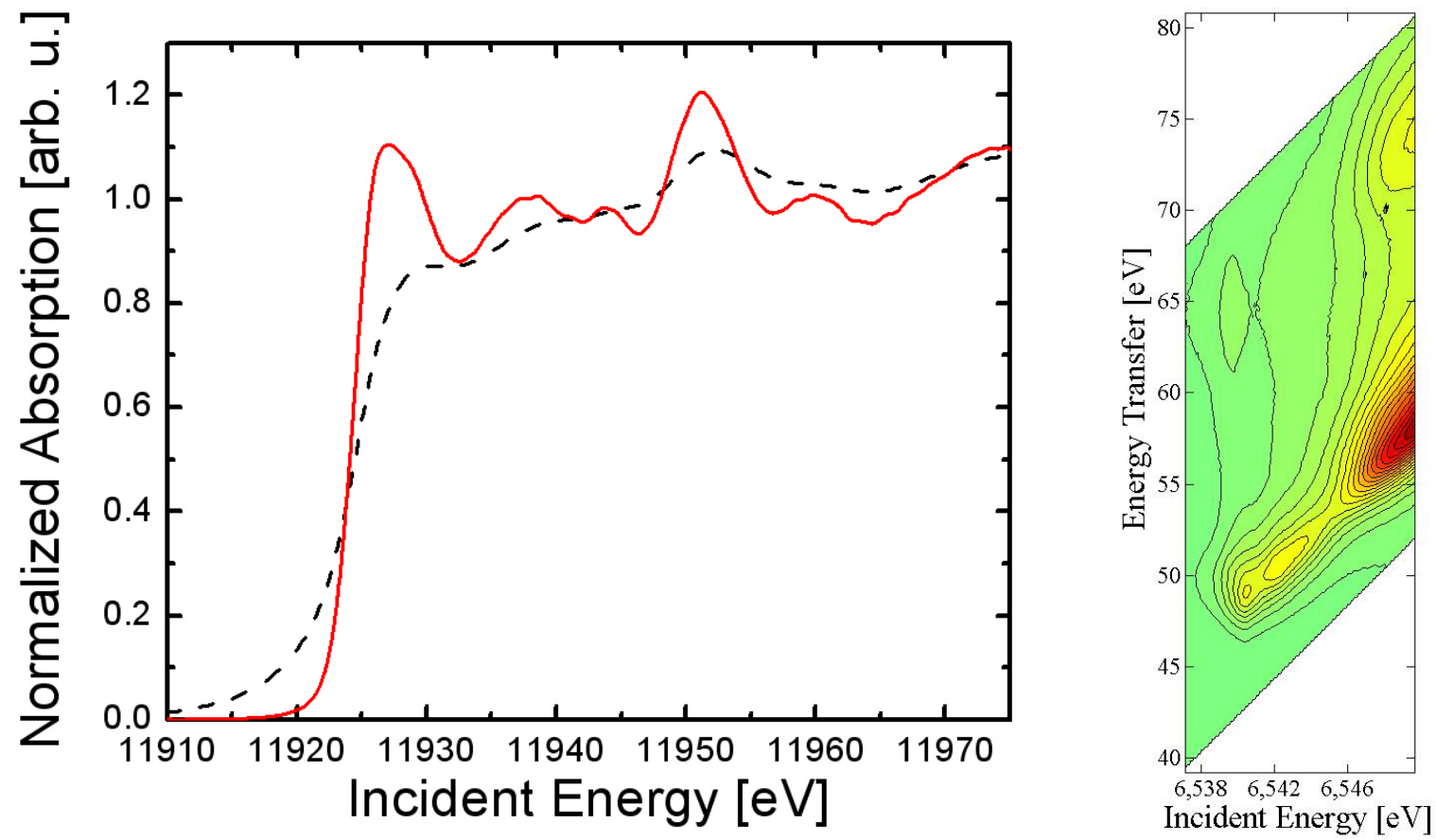

FIGURE 2. left: The $\mathrm{L}_{3}$ absorption edge of a Au foil detected in transmission mode (dotted) and using the HERFD technique (solid). Right: 1s3p RIXS plane of $\mathrm{Mn}_{2} \mathrm{O}_{3}$. The spin-down and spin-up directions occur at around $50 \mathrm{eV}$ and $65 \mathrm{eV}$ energy transfer, respectively. The spectra were recorded on beamline ID26 of the European Synchrotron Radiation Facility (ESRF).

The fact that HERFD XAS fully separates most fluorescence lines can be used to record extended absorption scans beyond unwanted edges that occur either in transmission or medium energy resolution fluorescence detection mode. The undesired edge may arise either from another element present in the sample or actually from the same element that is being studied. An example for the former is Fe in protein samples where the active site containing Mn is studied. The Fe K-edge occurs in the Mn EXAFS range and limits the available k-range for EXAFS analysis. Using HERFD XAS the Fe K-edge can be eliminated and the Mn EXAFS analysis greatly improved. The technique was successfully applied to study the oxygen evolving complex in the photosynthesis multi-protein complex photosystem II (PS II).[8] The same principle was applied for rare earths to eliminate the $\mathrm{L}_{1}$-edge in the $\mathrm{L}_{2}$ EXAFS.[9] However, here the pathways of possible electronic transitions are more complex and difficulties were encountered caused by cascade decays. We note that the constraint of having a dilute sample applies to HERFD as it applies to standard fluorescence detected XAS. 


\section{RIXSS}

The incident energy can be tuned to an absorption feature near the Fermi level that arises from an excitation into a localized state. If the radiative decay of this resonantly excited state is detected with lifetime resolution one can refer to resonant inelastic x-ray scattering spectroscopy (RIXSS). Figure 2 shows the 1s3p RIXS plane of $\mathrm{Mn}_{2} \mathrm{O}_{3}$. A 1 s electron is resonantly excited into the $\mathrm{K}$ absorption pre-edge and the subsequent $3 p$ to 1 s decay is monitored using the emission spectrometer.[3]

For a meaningful analysis of the RIXS spectra it is very helpful to record a full RIXS plane, i.e. cover the full range of possible combinations of incident and emitted energies as shown in Fig. 2. A line plot will only show the intensity variations in one direction. However, for the RIXS process intensity variations in two directions form a peak or spectral feature and interpretation of simple line plots is very difficult.

Experimentally the RIXS plane is recorded by fixing the emission energy and performing fast or continuous scans with the incident energy monochromator. This corresponds to diagonal scans in the 1s3p RIXS plane. To build up the RIXS plane the emission energy is changed step wise. A full RIXS plane can thus be recorded in one hour on a concentrated sample. In case the sample shows damage due to the X-ray beam it is possible to change the beam position on the sample with every change of emission energy. It is then necessary to correct the intensity for changes in the sample concentration or thickness.

RIXS spectroscopy greatly facilitates analysis of the $\mathrm{K}$ absorption pre-edges in transition metals because the lifetime of the final state and not the 1s excited state limit the resolution of the spectral features. It can be used to analyze valence shell spin and oxidation states as well as a diagnostic of the local symmetry.[10,11] The pre-edge can also be deconvoluted into spin-up and spin-down excitations which provides a stringent test of the theoretical model that aims to simulate the spectral shape.[12]

\section{CONCLUSIONS}

X-Ray emission detection with lifetime resolution is a technique complementary to XAS. An experimental setup should therefore ensure that both techniques can be applied simultaneously where the XAS is measured either in transmission or in fluorescence detection mode. Sample characterization on an X-ray beamline will then be more complete and the data analysis will be more robust because the different spectra will also serve to countercheck each other. X-ray emission spectrometers are currently being developed at various synchrotron radiation sources. It is desirable that these endstations will be installed permanently on a XAS beamline in order to minimize setup time and to make the technique readily available for users. With increasing user-friendliness in the operation, more and more user groups will appreciate the benefits of XES and it is realistic to assume that XES will become a standard technique.

\section{ACKNOWLEDGMENTS}

We would like to thank Dr. Frank F. M. de Groot for fruitful discussions. The ESRF support groups are gratefully acknowledged.

\section{REFERENCES}

1. EXAFS Spectroscopy. Techniques and Application, edited by B. K. Teo and D. C. Joy, New York, Plenum Press, 1981

2. X-ray Absorption: Principles, Applications, Techniques of EXAFS, SEXAFS, and XANES, edited by D. C. Koningsberger and R. Prins, New York, John Wiley \& Sons, 1988

3. P. Glatzel and U. Bergmann, Coord. Chem. Rev. 249, 65 (2005).

4. U. Bergmann, O. C. Mullins, and S. P. Cramer, Anal. Chem. 72 (11), 2609 (2000).

5. U. Bergmann, C. R. Horne, T. J. Collins et al., Chemical Physics Letters 302 (12), 119 (1999).

6. P Glatzel, U Bergmann, F. M. F. de Groot et al., in X-Ray and Inner-Shell Processes, edited by A. Bianconi and A. Marcelli (American Institute of Physics, Rome, 2002), Vol. 652, pp. 250.

7. J. A. van Bokhoven, C. Louis, J. T. Miller et al., Angewandte Chemie - International Edition in English 45, 4651 (2006).

8. J. Yano, Y. Pushkar, P. Glatzel et al., J. Am. Chem. Soc. 127 (43), 14974 (2005).

9. P. Glatzel, F. M. F. de Groot, O. Manoilova et al., Phys. Rev. B 72 (1) (2005).

10. P. Glatzel, U. Bergmann, W. W. Gu et al., J. Am. Chem. Soc. 124 (33), 9668 (2002).

11. P. Glatzel, U. Bergmann, J. Yano et al., J. Am. Chem. Soc. 126 (32), 9946 (2004).

12. X. Wang, F. M. F. deGroot, and S. P. Cramer, Physical Review B 56 (8), 4553 (1997). 